\title{
Demo Abstract: Using LEDs for Visible Light Communication and as a Wake-up Mechanism in the Internet of Things
}

\author{
Edgar Ripoll Vercellone \\ Universitat Politècnica de Catalunya \\ Idneo Technologies S.L. \\ edgar.ripoll.vercellone@upc.edu \\ Jordi Aubert \\ Idneo Technologies S.L. \\ jaubert@idneo.com
}

\author{
Vicent Ferrandiz \\ Idneo Technologies S.L. \\ vicent.ferrandiz@idneo.com
}

\author{
Manel Gasulla \\ Universitat Politècnica de Catalunya \\ manel.gasulla@upc.edu
}

\begin{abstract}
The design and implementation of autonomous wireless nodes for the Internet of Things (IoT) require paying exceptional attention to their sources of energy consumption in order to extend their battery lifetime. These nodes spend most of the time in standby mode and they only wake up to perform a quick defined task, usually sensing and measuring or transmitting information. In this demo, LEDs are used for Visible Light Communication (VLC). A low-cost, small-size and low-power consumption LED-based interface circuit connected to an $\mathrm{I} / \mathrm{O}$ microcontroller pin is proposed and tested. Moreover, this circuit also operates as a wake-up mechanism, aiding to achieve longer standby time and thus lower energy consumption. Our experiments are shown in actual commercial applications developed for a European project.
\end{abstract}

\section{CCS CONCEPTS}

- Hardware $\rightarrow$ Sensors and actuators; Wireless devices; $\bullet$ Networks $\rightarrow$ Short-range networks; Mobile networks; Wireless access networks;

\section{KEYWORDS}

Internet of Things, Visible Light Communication, Light Emitting Diodes, Energy Efficiency, Wake-up Mechanism.

\section{ACM Reference format:}

Edgar Ripoll Vercellone, Vicent Ferrandiz, Jordi Aubert, and Manel Gasulla. 2017. Demo Abstract: Using LEDs for Visible Light Communication and as a Wake-up Mechanism in the Internet of Things. In Proceedings of $A C M$ Sensys 2017, Delft, The Netherlands, November 2017 (Sensys'17), 2 pages. https://doi.org/

\section{INTRODUCTION}

The Internet of Things is a trending topic these days, which proposes that every existing device or object (machines, consumer goods, vehicles, etc.) is able to gather and transmit information

Permission to make digital or hard copies of part or all of this work for personal or classroom use is granted without fee provided that copies are not made or distributed for profit or commercial advantage and that copies bear this notice and the full citation on the first page. Copyrights for third-party components of this work must be honored.

For all other uses, contact the owner/author(s).

Sensys'17, November 2017, Delft, The Netherlands

(C) 2017 Copyright held by the owner/author(s).

ACM ISBN 978-x-xxxx-xxxx-x/YY/MM.

https://doi.org/ without human intervention. This is affecting people's daily lives and generating a new business area for companies. For example, in the Health area, many wearable sensors are being developed, such as glasses, wristbands, and hearing aids. In the Smart Society field we find utility meters, secure authentication wearables and electronic seals or locks. And in the Mobility and Production areas, some examples are industrial assets monitoring, indoor localization and navigation support and autonomous condition monitoring. In many of these applications, wireless sensors nodes are required. This work is carried out within the European project "EnSO" (Energy for Smart Objects) [1], where some requirements are sought for the sensor nodes of the IoT; for instance, low cost, small size and low-power consumption. Paticularly, the use of LEDs can help fulfill those requisites. Here, a LED will be used in a sensor node for wireless communication purposes, as well a wake-up mechanisms when the node is in sleep mode.

\section{USE OF THE LED AS EMITTER AND RECEIVER}

\subsection{Related Work}

In the nodes of the IoT, wireless communication usually takes place through radiofrecuency $(\mathrm{RF})$ waves. Different standards are used, such as NFC, Bluetooth Low Energy, Zigbee, etc. However, these solutions need an antenna and a transceiver. As a consequence, they add size, power consumption and cost to the nodes. As an alternative, in applications where line-of-sight and short range between the emitter and receiver exists, VLC using LEDs can be a feasible solution, particularly if the sensor node needs one or more LEDs for signaling purposes anyway.

The use of LEDs both as a light emitter and as receiver has been proposed in the literature, although not specifically for the nodes of the IoT requiring an ultra-low power consumption. For example, in [2], a two-way LED communication was developed. In order to detect light, the microcontroller had to be awakened periodically, precharge the parasitic LED capacitance and discharge it via the photogenerated LED current. This process wastes too much energy. In [3], a dual-mode passive driver circuit is described. But, in this case, the usage of LEDs both for emission and for reception involves the microcontroller's ADC, which implies an excessive power consumption. 


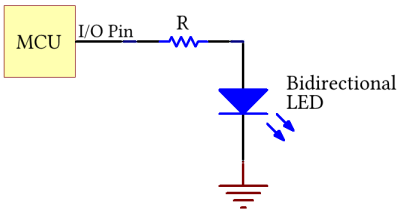

Figure 1: Circuit setup for LED bidirectional operation.

\subsection{Description of the LED Circuit}

Figure 1 shows the circuit we propose for VLC, where a LED is driven directly by a single microcontroller I/O pin. When the pin is configured as an output the LED acts as emitter. Contrariwise, when the pin is configured as a (high-impedance) input, the LED operates at photovoltaic mode (unbiased) and acts as a light receiver. Whenever a beam of light hits the LED a voltage builds up, whose level can be enough to be considered as a logic ' 1 '. Otherwise, a logic ' 0 ' is read in absence of light.

Therefore, in spite of the simplicity of the circuit, a bidirectional communication using a LED can be established just by changing the microcontroller pin setting from output (LED as emitter) to input (LED as receiver). Futhermore, configuring the input as an external interruption, a contactless wake-up mechanism is created. So, the MCU can remain at its lowest power mode until a wake up light signal arrives and, afterwards, start a bidirectional communication. Hence, the microcontroller no longer has to wake up periodically to check whether a communication has to be set up. This leads to a significant reduction of the consumed energy since the time in standby mode is prolonged. In some applications, several months can pass between the startup of the sensor node and its first wireless communication. Besides, by using a single LED instead of an RF-based communication, a low-price and small area solution is achieved.

\section{DEMONSTRATION}

The Figure 2 shows prototypes built for the EnSO project. The demonstration consists in the implementation of the above mentioned VLC and the wake-up mechanism using the circuit of Figure 1 . This will be tested on the prototypes (sensor nodes) in three real use cases:

- Electronic Seal: Monitors and records tampering attempts.

- Meter Supervisor: Residential natural gas meter.

- Smart Lock: Keeps track of accesses and allows the creation of different kinds of hierarchy profiles.

In all of them, the external light source that communicates with the LED of Figure 1 will be a smartphone flash. The node will be at first in standby mode. Light codes will be sent from the smartphone flash to the LED of the sensor node to first wake up the node and then to send information. The node will also use the LED as an indicator once received the wake up signal and the messages. For example, in the Smart Lock demonstration, the LED will emit a specific light codification indicating whether the access code previously emitted from the smartphone was successful or not. For demonstration purposes, the sensor node will also be connected to a laptop through an USB cable. Depending on the application and

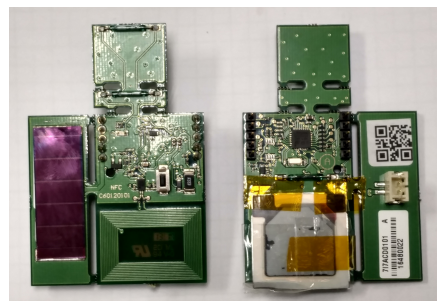

Figure 2: EnSO prototypes designed for testing several technologies; such as NFC, VLC, thin film batteries, etc.

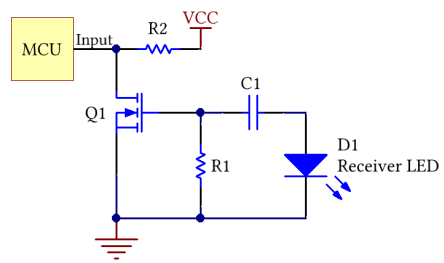

Figure 3: Interface circuit proposed to avoid high current consumption in case a constant light hits the LED.

the message code sent, a specific answer will be displayed in the laptop screen. A demo example can be seen at https://drive.google. com/file/d/0B-RraKj2Ifn_d0dIVjZ6YmZudXc/view?usp=sharing.

\section{FUTURE WORK}

The circuit simplicity of the Figure 1 has a downside. When a microcontroller $\mathrm{I} / \mathrm{O}$ pin is configured as an input, current consumption is significant whenever the input voltage is around the transition between the logic levels. This may be due, for example, to the presence of a constant light beam, e.g. ambient light coming from artificial lights or from the sun.

In Figure 3 a interface circuit is proposed to solve that problem. It consists in a monostable multivibrator. It blocks low frequency signals and generates pulses (to the input pin of the microcontroller) when the LED receives a frequency modulated light signal, being the time lenght of the pulses determined by $\mathrm{R}_{1}$ and $\mathrm{C}_{1}$. Therefore, if a constant light hits the LED, zero current is drawn from the power supply.

Work is in progress regarding the implementation of the circuit of Figure 3 alongside with a bidirectional communication feature.

\section{ACKNOWLEDGMENTS}

This work was supported by EnSO (Energy for Smart Objects), Grant Agreement No.: 692482, Idneo Technologies S.L. and the Secretariat of University and Research of the Ministry of Business and Knowledge of the Government of Catalonia.

\section{REFERENCES}

[1] 2017. Enso - Energy for Smart Objects. (2017). http://www.enso-ecsel.eu/

[2] Paul Dietz, William Yerazunis, and Darren Leigh. 2003. Very Low-Cost Sensing and Communication Using Bidirectional LEDs. Springer Berlin Heidelberg, Berlin, Heidelberg, 175-191. https://doi.org/10.1007/978-3-540-39653-6_14

[3] S. Li, A. Pandharipande, and F. M. J. Willems. 2016. Daylight Sensing LED Lighting System. IEEE Sensors fournal 16, 9 (May 2016), 3216-3223. https: //doi.org/10.1109/JSEN.2016.2520495 